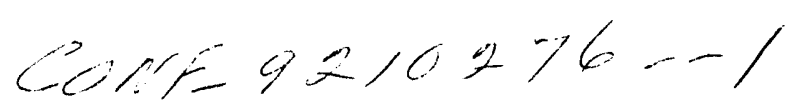

\title{
Nanophase Materials Assembled from Atom Clusters*
}

Richard W. Siegel

Materials Science Division

Argonne National Laboratory

Argonne, IL 60439

$\mathrm{ANL} / \mathrm{MSD} / \mathrm{CP}--76871$

DE93 005559

\section{December 1992}

The slbmitted manuscript has been authored by
a contractor of the U.S. Government under
contract No. W-31-109ENG-38. Accordingly.
the U.S. Government retains a nonexclusive.
royalky-tree license to publish or reproduce the
published form of this contribution, or allow
others to do so, for U.S. Government purposes.

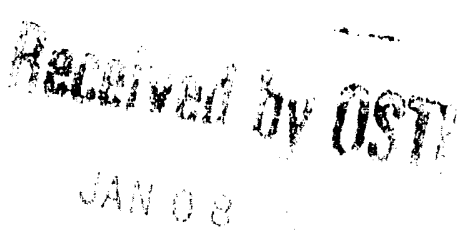

INVITED paper presented at The 4th NEC Symposium on Fundamental Approaches to New Material Phases: Physics and Chemistry of Nanometer-Scale Materials, 11-15 October 1992, Karuizawa, Japan; to be published in Materials Science \& Engineering B (1993).

*Work supported by the U. S. Department of Energy, BES-Materials Sciences, under Contract W-31-109-Eng-38. 
Invited paper presented at the 4th NEC Symposium on Fundamental Approaches to New Material Phases: Physics and Chemistry of Nanometer-Scale Materials, 11-15 October 1992; to be published in Materials Science \& Engineering B (1993).

\title{
Nanophase materials assembled from atom clusters
}

\author{
Richard W. Siegel \\ Materials Science Division, Argonne National Laboratory, Argonne, Illinois 60439 USA
}

(Received November 28, 1992)

\begin{abstract}
Nanometer-scale atom clusters (with average diameters below $20 \mathrm{~nm}$ ) of a variety of materials, including both metals and ceramics, have been synthesized by precursor evaporation and condensation in high-purity gases. The gas-entrained clusters can be collected and subsequently consolidated in situ under ultrahigh vacuum or other controlled atmosphere conditions to create bulk nanophase materials. These ultrafine-grained materials have properties that are often significantly different and considerably improved relative to those of their coarser-grained counterparts. The observed property changes relate to both their small grain sizes and the large percentage of their atoms in grain boundary environments. Since it is becoming apparent that their properties can be engineered during gas-phase synthesis and subsequent processing, nanophase materials assembled from atom clusters should have significant potential for technological development in a variety of applications. Some of the recent research on nanophase materials is reviewed.
\end{abstract}




\section{Introduction}

Interest in a wide variety of synthetic nanostructured materials, with average grain or other structural domain sizes below $100 \mathrm{~nm}$, has increased during the past several years with the anticipation that their properties will be different from, and often superior to, those of conventional materials that have phase or grain structures on a coarser size scale [1]. This interest has been recently enhanced by the efforts and successes in synthesizing a variety of beautifully symmetric and captivating atom clusters, such as those derived from carbon discussed in this Symposium. However, the interest in this class of new materials was earlier stimulated by work on a variety of zero-dimensionality quantum-well structures, one-dimensionally modulated multilayered materials with nanometer scale modulations, and three-dimensionally modulated, bulk nanophase materials via the assembly of clusters of atoms [2]. This latter class of materials is of particular interest with respect to the so-called "fullerenes", since it should become possible in the future to synthesize bulk nanophase materials with unique properties by the controlled assembly of these carbon clusters and tubules either in single phase ensembles or, together with other types of clusters, in multicomponent composite materials.

It should be made clear that cluster-assembled nanophase materials are only one of the broad class of nanostructured materials artificially synthesized with microstructures modulated in zero to three dimensions on length scales less than $100 \mathrm{~nm}$ that it has become possible to create over the past few years. It is important to recognize, however, that the various types of nanostructured materials share three features, atomic domains spatially confined to less than $100 \mathrm{~nm}$, significant atom fractions associated with interfacial environments, and interactions between their constituent domains, that determine their unique properties. The broad class of nanostructured materials, as indicated schematically in fig. 1, thus includes zero-dimensionality atom clusters (with any aspect ratio, including filaments or tubules), cluster assemblies, one- and two-dimensionally modulated multilayers and overlayers (or buried layers), respectively, and their three-dimensional analogues, nanophase materials [3]. 
Atom clusters in the nanometer s.... regime, containing hundreds to tens of thousands of atoms, of a wide varity of materials can now be produced in sufficient numbers by means of either physical or chemical processes that they can be assembled into bulk materials that can be investigated by a variety of conventional experimental methods. These nanophase materials can take advantage of and incorporate a number of size-related effects in condensed matter ranging from electronic effects (so-called "quantum size effects") caused by spatial confinement of delocalized valence electrons and altered cooperative ("many body") atom phenomena, such as lattice vibrations or melting, to the suppression of such lattice-defect mechanisms as dislocation generation and migration in confined grain sizes. The possibilities to assemble size-selected atom clusters into new materials with unique or improved properties may thus revolutionize our ability to engineer a wide variety of controlled optical, electronic, mechanical, and chemical properties with attendant useful technological applications.

\section{Synthesis and structure}

The synthesis of a nanophase material, or any other nanostructured material, from atomic or molecular precursors depends upon the ability to control a variety of microscopic aspects of the condensed ensemble. The most important of these are (1) the size and size distribution of the constituent phases or structures, (2) the composition of the constituent phases, and (3) the nature of the interfaces created between constituent phases and, hence, the nature of the interactions across the interfaces. The desirable domain sizes are generally below $100 \mathrm{~nm}$, since it is in this size range that various properties begin to change significantly owing to a variety of confinement effects. A property will be altered when the entity or mechanism (or combination thereof) responsible for that property is confined within a domain size smaller than some critical length associated with that entity or mechanism. Control of the composition of the constituent phases can simply mean maintaining phase purity during synthesis in a single phase material, such as an oxide or a metal, or it can mean controlling the impurity doping levels, the stoichiometries, the solute gradients, the phase mixtures, or combinations of these in more complex nanophase materials. 
However, the nanometer length scales over which such composition control must be maintained can push the limits of our technical capabilities. It is the interplay among these three features (size, composition, and interfaces) that determines the properties of nanostructured materials.

Discussion of the very wide variety of synthesis and processing methods for the creation of nanostructured materials is well beyond the scope of the present paper. A more comprehensive treatment appears elsewhere [4] and previous reviews [1,5] can also be usefully consulted in this regard. In the present paper, tnanophase materials assembled from atom clusters are used as a representative example of some of the capabilities of creating new materials by assembling matter on a nanometer scale. There are a number of advantages associated with the synthesis of materials from atom clusters. Some of these stem from the nanometer scale of the structures assembled and others arise from the inherent flexibility of dealing with clusters as the "building blocks" of these materials. Two examples of the manner in which material properties can be varied through the assembly of constituent domains through the effects of domain interaction are usful to consider in this regard.

The first example relates to the optical absorption behavior of CdS clusters with diameters in the nanometer size regime made by any of a variety of methods, including chemical precipitation in solutions [6] or in zeolite supports [7]. This behavior is rather different from that for bulk CdS. The absorption edge is blue shifted to appreciably shorter wavelengths, owing to the the effects of quantum confinement where the size of the cluster becomes comparable to and smaller than the effective size of the excitonic state responsible for this absorption. However, when these clusters are synthesized in zeolite supports with increasing loading, such that they become close enough to begin to interact through quantum tunneling, the absorption edge begins to shift back toward bulk behavior [8]. Hence, control of the average distance between clusters, even though they are not actually in contact, being separated by the cage-like structure of the zeolite, can enable control over a property of the assembled cluster ensemble.

The second example is based upon a hypothetically simple multilayered nanostructure, an alternating set of uniform thickness $\mathrm{Cu}$ layers separated only by twist grain boundaries with twist axis normal to the layers and boundaries. Calculations [9] of the variation of the average lattice parameters and elastic constants of this chemically homogeneous $\mathrm{Cu}$ multilayer with decreasing 
multilayer modulation wavelength demonstrate that even for such a simple case of layer-layer interaction, the effects (up to $20 \%$ at the smallest wavelengths) on the elastic properties can be rather significant in the nanometer regime. One can expect that in real nanostructured systems, the effects of such domain interactions may result in even more interesting property manifestations.

An important aspect of materials with reduced spatial dimensions in the nanometer size regime that has to date inspired little attention is phase stability. As one reduces the sizes of constituent phases sufficiently, equilibrium phase relations will change owing to expected changes in a variety of electronic and thermodynamic parameters of the confined atomic system. For example, it has long been known [10-12] that the melting temperature of small metal clusters is suppressed well below that for the bulk solid, owing in part to a higher effective pressure in the confined system resulting from the Gibbs-Thomson effect. This has also been recently demonstrated [13] for 2.4-7.6 $\mathrm{nm}$ diameter CdS semiconductor clusters synthesized by the inverse micelle chemical precipitation method [6]. 'The melting-point suppression can be rather dramatic; the smallest $\mathrm{CdS}$ clusters were observed to melt at about $1000 \mathrm{~K}$ below the bulk melting temperature. Related effects are also seen in cluster-consolidated materials. Investigation of nanophase Er oxides synthesized by the gas-condensation route showed [14] that new oxide phases related to previously observed high-pressure structures were formed in grains of about $10 \mathrm{~nm}$ diameter, even though the samples had never been exposed to sufficiently high external pressures. Similarly, it was recently reported [15] that nanophase $(8 \mathrm{~nm})$ yttria can be stabilized in its high-pressure polymorphic structure, $\gamma-\mathrm{Y}_{2} \mathrm{O}_{3}$. Thus, it seems clear that the effects of reduced spatial dimensions on phase stability need further investigation, since one cannot expect that the phase equilibria we know for conventional materials systems will apply in general to those of significantly reduced dimensions.

The synthesis of nanophase materials via the consolidation in vacuum of gas-condensed clusters has been described in detail elsewhere [16]; it is sufficient to give only a brief outline of the process here. A precursor material, either metal or compound, is evaporated in a gaseous atmosphere maintained at a few hundred $\mathrm{Pa}$ pressure in a back-filled, ultrahigh-vacuum chamber. The evaporated atoms or molecules lose energy via collisions with the gas atoms or molecules and undergo a homogeneous condensation to form atom clusters in the highly supersaturated vicinity of the precursor source [17], as indicated schematically in fig. 2. In order to maintain small cluster 
sizes, by preventing further atom or molecule accretion, the clusters once nucleated must be removed rapidly from the region of high supersaturation. Since the clusters are already gas entrained, this is easily accomplished by moving the condensing gas. Such gas motion has generally resulted from natural convection under the action of gravity and the temperature difference between the precursor source and a thermophoretic cluster collector cooled by liquid nitrogen. However, a forced gas flow can also be used, and with significant advantage in terms of cluster size control and process efficiency. The clusters are then removed from the collector under vacuum and consolidated at room temperature at pressures up to about 1-2 GPa by means of a piston-and-anvil device. The resulting samples are usually about $1 \mathrm{~cm}$ in diameter and about 0.1-0.5 mm thick, depending upon the amount of precursor material evaporated and the experiment planned. A typical evaporation in the laboratory now takes of order of about $1 \mathrm{~h}$ to make such a sample; fortunately, the process can be (and has been) readily scaled up by several orders of magnitude.

The clusters that are collected on the surface of the collector, and which subsequently become the grains of the consolidated polycrystalline nanophase aggregate (shown in the STM image [18] in fig. 3), have a rather narrow size distribution, usually with a full-width at half-maximum of about $\pm 25 \%$ of the peak diameter, and the log-normal shape typical of clusters formed via gas-condensation [17]. This shape is rather typical for the grain size distribution in any of the nanophase materials thus far produced by this method. Transmission electron microscopy [19-22] has shown that the grains in nanophase compacts are essentially equiaxed, similar to the atom clusters from which they were formed. On the other hand, the observations that the densities of nanophase materials consolidated from initially equiaxed clusters extend well beyond the theoretical limit $(78 \%)$ for close packing of identical spheres indicate that an extrusion-like deformation of the clusters must result during the consolidation process, filling in (at least partially) the pores among the grains. However, essentially all of the nanophase materials consolidated from clusters at room temperature to date have invariably posessed a degree of porosity ranging from about $25 \%$ to less than $5 \%$, as measured by Archimedes densitometry, with the larger values for ceramics and the smaller ones for metals. Evidence for this porosity was first obtained by positron annihilation spectroscopy $[19,23,24]$ and more recently by precise densitometry [25] and porosimetry [26, 
27] measurements. These measurements have together shown that the porosity in as-consolidated nanophase metals and ceramics is primarily in the less than $100 \mathrm{~nm}$ size regime (although some larger porous flaws have been observed), and frequently of comparable sizes to the grains, but that the porosity is to a great extent interconnected and intersects with the specimen surfaces. Fortunately, it appears now that this porosity can be controlled during synthesis and can be removed during consolidation at elevated temperatures without sacrificing the ultrafine grain sizes in these materials.

An important aspect of nanophase materials assembled from atom clusters is their apparently inherent stability against grain growth $[28,29]$. Their grain sizes generally remain rather deeply metastable to elevated temperatures until about 0.4 to 0.5 of the absolute melting temperature is reached in single-phase nanophase materials. This stability (actually, deep metastability) is rather typical for the nanophase oxides investigated and for nanophase metals also. It appears that the narrow grain size distributions in these cluster-assembled materials coupled with their relatively flat and faceted grain boundaries [30] (and also enhanced by their multiplicity of grain boundary junctions) place these nanophase structures in a local minimum in energy from which they are not easily extricated. They are thus analogous to a variety of closed-cell foam structures, which are stable despite their large stored surface energy. Under such conditions, only at temperatures above which bulk diffusion distances are comparable to or greater than the mean grain size will this metastability give way to global energy minimization via rapid grain growth. One can, of course, intentionally stabilize against grain growth by appropriate doping or composite formation in the grain boundaries to prevent or to retard their migration.

\section{Properties}

Cluster-assembled nanophase materials, as other nanostructured materials, have properties that are significantly different and frequently improved in comparison with those of conventional coarser-scale structures. These result from the impact of various combinations of the three critical aspects (size, composition, and interfaces) of their nature cited in Sec. 2. Some examples from 
this particular class of nanostructured materials will be presented here to attempt to give a sense of how the interplay among these aspects can alter useful technological properties of these materials.

The processing of nanophase materials is dramatically improved by the combination of ultrafine cluster (grain) sizes, short diffusion distances, and cleanliness of cluster surfaces and interfaces prior to and after consolidation, respectively. For example, nanophase $\mathrm{TiO}_{2}$ (rutile) exhibits significant improvements in both sinterability and resulting mechanical properties relative to conventionally synthesized coarser-grained rutile $[19,26,31,32]$. Nanophase $\mathrm{TiO}_{2}$ with a 12 $\mathrm{nm}$ initial mean grain diameter has been shown [19] to sinter under ambient pressures at up to $600^{\circ} \mathrm{C}$ lower temperatures than conventional coarser-grained rutile, and without the need for any compacting or sintering additives. Furthermore, sintering the same nanophase material under pressure (at $1 \mathrm{GPa}$ ), or with appropriate dopants such as $\mathrm{Y}$, can further reduce the sintering temperatures while suppressing grain growth as well, thus allowing for the unique possibility to sinter nanophase ceramics to full density while retaining their ultrafine grain size [26]. Also, atomic diffusion in nanophase materials has been found to be very rapid compared with conventional materials. Measurements of self- and solute-diffusion [31, 33-36] in as-consolidated nanophase metals and ceramics indicate that atomic transport is orders of magnitude faster in these materials than in coarser-grained polycrystalline samples. While this very rapid diffusion appears to be intrinsically coupled with the porous nature of the many interfaces in these materials [31], there exist unique possibilities for efficiently doping nanophase materials at relatively low temperatures to synthesize materials with tailored optical, electrical, or mechanical properties.

The most striking mechanical property change resulting from reducing the grain sizes of nanophase metals is the significant increase in their strength $[26,37,38]$. Figure 4 shows microhardness results for several samples of nanophase $\mathrm{Cu}$ compared with results for a coarser-grained sample. The smallest grain size $(6 \mathrm{~nm})$ sample exhibits about a $500 \%$ increase in hardness over the coarser-grained (ca. $50 \mu \mathrm{m}$ ) sample and effects of similar magnitude have been observed for nanophase $\mathrm{Pd}$ as well [37]. The common strengthening behavior found in cluster-assembled nanophase $\mathrm{Cu}$ and $\mathrm{Pd}$ indicates that this response is generic to nanophase metals, a conclusion that is supported by observations $[39,40]$ that nanophase metals and alloys produced via mechanical attrition (which yields similar nanometer-scale grains) also exhibit 
significantly enhanced strength. It thus appears that grain confinement is the dominant cause for the increased strength of nanophase metals, although an additional contribution to the observed strengthening may result from elastic strain accommodation [25] arising from cluster consolidation or mechanical attrition. Metals, which are coventionally ductile owing to the usual ease in creating and moving dislocations through their crystal lattices, will become significantly harder when grain sizes are reduced to the point where dislocation sources are no longer able to operate at low levels of applied stress. Since the stress to operate a common Frank-Read dislocation source is inversely proportional to the spacing between dislocation pinning points, the critical confinement length here is that spacing for which the stress to operate this source becomes larger than the conventional yield stress for the given metal.

In contrast to the mechanical behavior of nanophase metals, it has been found that nanophase ceramics are easily formed [19,41-43], significantly more so than conventional ceramics which are very stong but brittle. Nanoindenter measurements on nanophase $\mathrm{TiO}_{2}$ [32] and $\mathrm{ZnO}$ [44] have demonstrated that a dramaic increase of strain rate sensitivity occurs with decreasing grain size, as shown in fig. 5. Since a strong and similar grain-size dependence was found for both sets of samples (in which the porosity was different but changing very little with grain size), it appears that this increased tendency toward ductility is an intrinsic property of these ultrafine-grained ceramics. The strain rate sensitivity values at the smallest grain sizes investigated $(12 \mathrm{~nm}$ in nanophase $\mathrm{TiO}_{2}$ and $7 \mathrm{~nm}$ in $\mathrm{ZnO}$ ) indicate limited ductile behavior of these nanophase ceramics at room temperature, as well as significant potential for increased ductility at even smaller grain sizes and elevated temperatures where superplasticity of these materials may yet be observed. Limited evidence for such behavior has recently appeared [43]. The ductility of nanophase ceramics appears to result from increased grain boundary sliding, aided by the presence of porosity, ultrafine grain size, and probably rapid short-range diffusion as well. This behavior is therefore dominated by the presence of the numerous interfaces in these materials and the very short diffusion distances involved in effecting the necessary atomic healing of incipient cracks for grain boundary sliding to progress at the strain rates utilized without fracturing the sample. Extrapolating from this apparently generic behavior, one can expect that grain boundary sliding mechanisms, accompanied by short-range diffusion assisted healing events, would be expected to 
increasingly dominate the deformation of a wide range of nanophase materials. Enhanced forming and even superplasticity in a wide range of nanophase materials, including intermetallic compounds, ceramics, and semiconductors might become a reality. Consequently, increased opportunities for high deformation or superplastic near-net-shape forming of a very wide range of even conventionally rather brittle and difficult to form materials could result.

Control of the porosity or surface area of nanophase materials during synthesis can lead to a number of useful properties. For example, it was recently demonstrated [45] that the oxidation step in the synthesis of cluster-consolidated nanophase $\mathrm{Y}_{2} \mathrm{O}_{3}$ can be controlled so that the resulting "green-state" (as-consolidated) porosity of 25-35\% has a size distribution sufficiently small and narrow that the material is effectively transparent. The ability to reduce the size of the porosity in such material to well below the wavelengths of visible or other radiation, coupled with the possibilities for efficient doping of these materials cited previously, should lead to a variety of interesting optical properties and related applications. The chemical reactivity of nanophase materials, with their high surface areas compared to conventional materials, can also be rather striking. Since the gas-condensed, high-surface-area clusters are assembled via consolidation, an excellent degree of control can be exercised over the total available surface area in these intrinsically self-supported ensembles. Composition control can also be readily achieved. Measurements [46] of the decomposition of $\mathrm{H}_{2} \mathrm{~S}$ over lightly consolidated nanophase $\mathrm{TiO}_{2}$ at $500^{\circ} \mathrm{C}$ demonstrate the enhanced chemical reactivity of nanophase materials rather well. Figure 6 shows the catalytic activity for $\mathrm{S}$ removal from $\mathrm{H}_{2} \mathrm{~S}$ in a $\mathrm{N}_{2}$ gas stream at $500^{\circ} \mathrm{C}$ via dissociative adsorption for nanophase $\mathrm{TiO}_{2}$ (rutile) compared with that for a number of other commercially available forms of $\mathrm{TiO}_{2}$ having either the rutile or anatase structure. The cluster-assembled $\mathrm{TiO}_{2}$ was considerably more reactive than any of the other samples tested, both initially and also after extended exposure to the $\mathrm{H}_{2} \mathrm{~S}$. This greatly enhanced activity was shown to result from a combination of unique features of the nanophase $\mathrm{TiO}_{2}$, all of which could be controlled during its synthesis and processing, its high surface area combined with its rutile structure and its oxygen deficient composition $[47,48]$. While such results are rather limited at present. they indicate that the prospects for engineering functional nanophase materials via cluster assembly may be quite interesting. 


\section{Conclusions}

Tremendous opportunities are unfolding for creating nanostructured materials with new architectures at nanometer length scales from atomic or molecular precursors via the assembly of atom clusters and by a myriad of other techniques now becoming available $[49,50]$. The keys to the future of nanostructured materials, however, will be coth in our ability to significantly change for the better the properties of materials by artificially structuring them on these nanometer length scales and in developing the methods for producing these materials in commercially viable quantities. The future appears to hold great promise based upon the limited knowledge that has already been accumulated, but much work rems ns to be done.

The examples of cluster assembly presented in Sec. 4 have shown that in some cases at least it is already possible to dramatically alter a variety of technologically important properties of materials in this manner. The strength of normally very ductile pure metals can be dramatically increased simply by reducing their grain sizes into the range where dislocation generation and migration becomes confined and difficult. Normally brittle ceramics can be rendered more ductile and formable by reducing their grain sizes into the range where grain boundary sliding apparently becomes facilitated by the high number density of internal interfaces and the rapid atomic diffusion over nanometer length scales. Both the chemical reactivity of a high-surface-area self-supported nanophase catalyst and the optical transparancy of a nanophase ceramic can be greatly increased or even enabled by control of the cluster synthesis and assembly. The possibilities for engineering both the catalytic and optical properties of nanostructured materials will clearly be further enhanced by the ability to control the compositions and defect structures in these materials. Such examples suggest that even more exciting opportunities may be available as we learn to assemble more sophisticated multifunctional composite nanostructured materials in the future. 


\section{Acknowledgements}

This work was supported by the U.S. Department ur Energy, BES-Materials Sciences, under Contract W-31-109-Eng-38.

\section{References}

1. B. H. Kear, L. E. Cross, J. E. Keem, R. W. Siegel, F. Spaepen, K. C. Taylor, E. L. Thomas, and K.-iJ. Tu, Research Opportunities for Materials with Ultrafine Microstructures (National Academy, Washington, DC, 1989), Vol. NMAB-454.

2. R. P. Andres, R. S. Averback, W. L. Brown, L. E. Brus, W. A. Goddard, III, A. Kaldor, S. G. Louie, M. Moskovits, P. S. Peercy, S. J. Riley, R. W. Siegel, F. Spaepen, and Y. Wang, J. Mater. Res. 4, 704 (1989).

२. W. Siegel, Nanostructured Mater. 3, 1 (1993).

4. R. W. Siegel, in Mechanical Properties and Deformation Behavior of Materials Having Ultra-Fine Microstructures, M. A. Nastasi et al., eds. (Kluwer, Dordrecht, 1993) in press.

5. H. Gleiter, Progress in Materials Science 33, 223 (1989).

6. M. L. Steigerwald and L. E. Brus, Ann. Rev. Mater. Sci. 19, 471 (1991).

7. G. D. Stucky and J. E. Mac Dougall, Science 247, 669 (1990).

8. N. Herron, Y. Wang, M. Eddy, G. Stucky, D. E. Cox, K. Moller, and T. Bein, J. Amer. Chem. Soc. 111, 530 (1989).

9. D. Wolf and J. F. Lutsko, Phys. Rev. Lett. 60, 1170 (1988).

10. Ph. Buffat and J.-P. Borel, Phys. Rev. A 13, 2287 (1976).

11. M. Y. Han and R. L. Whetten, Phys. Rev. Lett. 61, 1190 (1988).

12. R. S. Berry, J. Jellinek, and G. Natansen, Phys. Rev. A 30, 919 (1984).

13. A. N. Goldstein, C. M. Echer, and A. P. Alivisatos, Science 256, 1425 (1992).

14. Z. Li, H. Hahn, and R. W. Siegel, Mater. Lett. 6, 342 (1988). 
15. G. Skandan, C. M. Foster, H. Frase, M. N. Ali, J. C. Parker, and H. Hahn, Nanostructured Mater. 1, in press (1992).

16. R. W. Siegel, in Materials Science and Technology, Vol. 15, R. W. Cahn, ed.(VCH, Weinheim, 1991) p. 583.

17. C. G. Granquist and R. A. Buhrman, J. Appl. Phys. 47, 2200 (1976).

18. K. Sattler, G. Raina, M. Ge, N. Venkateswaran, J. Xhie, Y. X. Liao, and R. W. Siegel, J. Appl. Phys., to be published (1993).

19. R. W. Siegel, S. Ramasamy, H. Hahn, Z. Li, T. Lu, and R. Gronsky, J. Mater. Res. 3, 1367 (1988).

20. G. J. Thomas, R. W. Siegel, and J. A. Eastman, Mater. Res. Soc. Symp. Proc. 153, 1' (1989).

21. G. J. Thomas, R. W. Siegel, and J. A. Eastman, Scripta Metall. et Mater. 24, 201 (1990).

22. W. Wunderlich, Y. Ishida, and R. Maurer, Scripta Metall. et Mater. 24, 403 (1990).

23. H. E. Schaefer, R. Würschum, M. Scheytt, R. Birringer, and H. Gleiter, Mater. Sci. Forum 15-18, 955 (1987).

24. H. E. Schaefer, R. Würschum, R. Birringer, and H. Gleiter, Phys. Rev. B 38, 9545 (1988).

25. G. W. Nieman, J. R. Weertman, and R. W. Siegel, J. Mater. Res. 6, 1012 (1991).

26. H. Hahn, J. Logas, and R. S. Averback, J. Mater. Res. 5, 609 (1990).

27. W. Wagner, R. S. Averback, H. Hahn, W. Petry, and A. Wiedenmann, J. Mater. Res. 6, 2193 (1991).

28. J. A. Eastman, Y. X. L.aa, A. Narayanasamy, and R. W. Siegel, Mater. Res. Soc. Symp. Proc. 155, 255 (1989).

29. R. W. Siegel, Mater. Res. Soc. Symp. Proc. 196, 59 (1990).

30. R. W. Siegel and G. J. Thomas, Ultramicroscopy 40, 376 (1992).

31. R. S. Averback, H. Hahn, H. J. Höfler, J. L. Logas, and T. C. Chen, Mater. Res. Soc. Symp. Proc. 153, 3 (1989).

32. M. J. Mayo, R. W. Siegel, A. Narayanasamy, and W. D. Nix, J. Mater. Res. 5, 1073 (1990). 
33. J. Horváth, R. Birringer, and H. Gleiter, Solid State Commun. 62, 319 (1987).

34. J. Horváth, Defect and Diffusion Forum 66-69, 207 (1989).

35. H. Hahn, H. Höfler, and R. S. Averback, Defect and Diffusion Forum 66-69, 549 (1989).

36. S. Schumacher, R. Birringer, R. Straub, and H. Gleiter, Acta Metall. 37, 2485 (1989).

37. G. W. Nieman, J. R. Weertman, and R. W. Siegel, Scripta Metall. 23, 2013 (1989).

38. G. W. Nieman, J. R. Weertman, and R. W. Siegel, Scripta Metall. et Mater. 24, 145 (1990).

39. J. S. C. Jang and C. C. Koch, Scripta Metall. et Mater. 24, 1599 (1990).

40. C. C. Koch and Y. S. Cho, Nanostructured Mater. 1, 207 (1992).

41. J. Karch, R. Birringer, and H. Gleiter, Nature 330, 556 (1987).

42. J. Karch and R. Birringer, Ceramics International 16, 291 (1990).

43. H. Hahn, J. Logas, H. J. Höfler, P. Kurath, and R. S. Averback, Mater. Res. Soc. Symp. Proc. 196, 71 (1990).

44. M. J. Mayo, R. W. Siegel, Y. X. Liao, and W. D. Nix, J. Mater. Res. 7, 973 (1992).

45. G. Skandan, H. Hahn, and J. C. Parker, Scripta Metall. et Mater. 25, 2389 (1991).

46. D. D. Beck and R. W. Siegel, J. Mater. Res. 7, 2840 (1992).

47. J. C. Parker and R. W. Siegel, J. Mater. Res. 5, 1246 (1990).

48. J. C. Parker and R. W. Siegel, Appl. Phys. Lett. 57, 943 (1990).

49. R. S. Averback, J. Bernholc, and D. L. Nelson, eds., Mater. Res. Soc. Symp. Proc. 206 (1991).

50. Nanostructured Mater. 3, in press (1993): Proc. First International Conference on Nanostructured Materials. 


\section{Figure captions}

Figure 1. Schematic of the four types of nanostructured materials [3], classified according to integral modulation dimensionality: zero - clusters of any aspect ratio from 1 to $\infty$; one - multilayers; two - ultrafine-grained overlayers or buried layers; three - nanophase materials. Intermediate dimensionalities can exist.

Figure 2. Conceptual model for the formation of atom clusters via gas condensation. After [17].

Figure 3. Scanning tunneling micrograph of nanophase Ag [18].

Figure 4. Vickers microhardness measurements at a number of positions across several nanophase $\mathrm{Cu}$ samples ranging in grain size from 6 to $50 \mathrm{~nm}$, compared with similar measurements from an annealed conventional $50 \mu \mathrm{m}$ grain size $\mathrm{Cu}$ sample [25].

Figure 5. Strain rate sensitivity of nanophase $\mathrm{TiO}_{2}$ [32] and $\mathrm{ZnO}$ [44] as a function of grain size. The strain rate sensitivity was measured by a nanoindentation method and the grain size was determined by dark-field transmission electron microscopy.

Figure 6. Activity of nanophase $\mathrm{TiO}_{2}$ for $\mathrm{H}_{2} \mathrm{~S}$ decomposition as a function of exposure time at $500^{\circ} \mathrm{C}$ compared with that from several commercially available $\mathrm{TiO}_{2}$ materials and a reference (A: $76 \mathrm{~m}^{2} / \mathrm{g}$ nanophase rutile; $\mathrm{B}: 61 \mathrm{~m}^{2} / \mathrm{g}$ anatase; $\mathrm{C}: 2.4 \mathrm{~m}^{2} / \mathrm{g}$ rutile; $\mathrm{D}: 30 \mathrm{~m}^{2} / \mathrm{g}$ anatase; $\mathrm{E}: 20$ $\mathrm{m}^{2} / \mathrm{g}$ rutile; F: reference alumina). From [46]. 


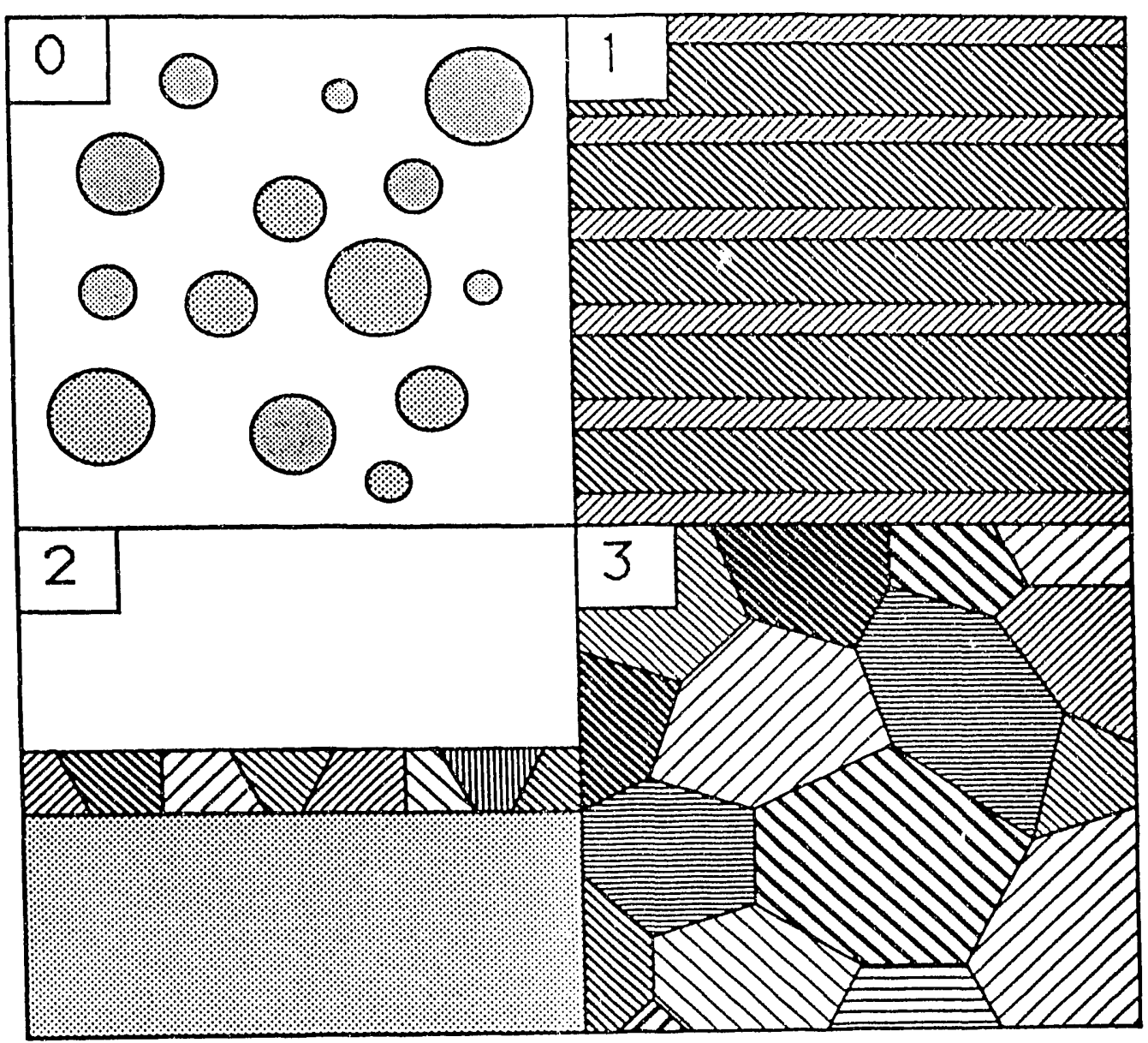

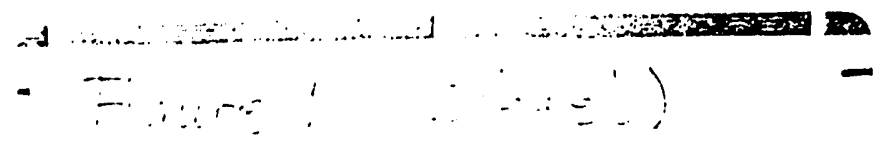




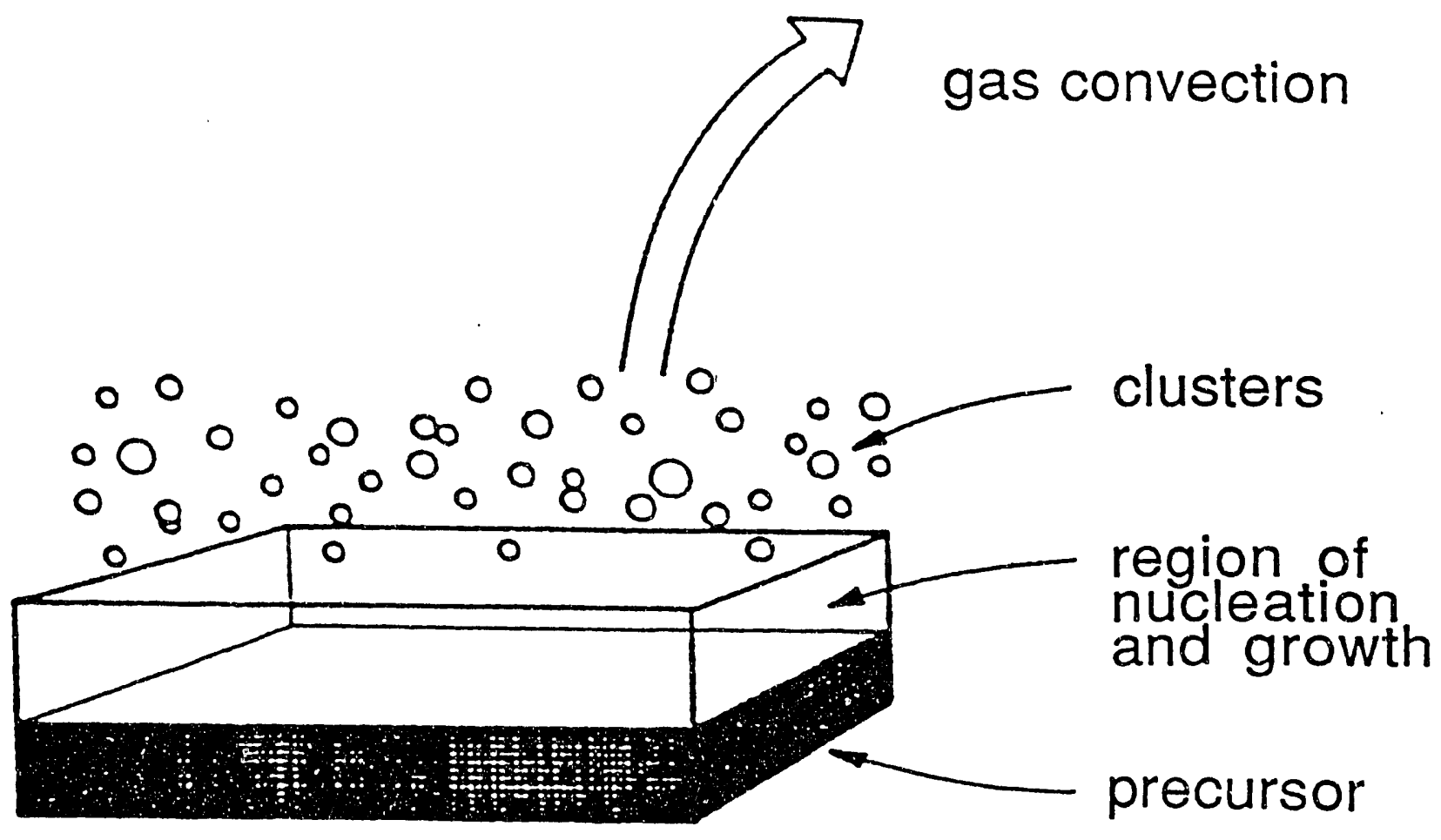

- Figure 2 (Siejel) 


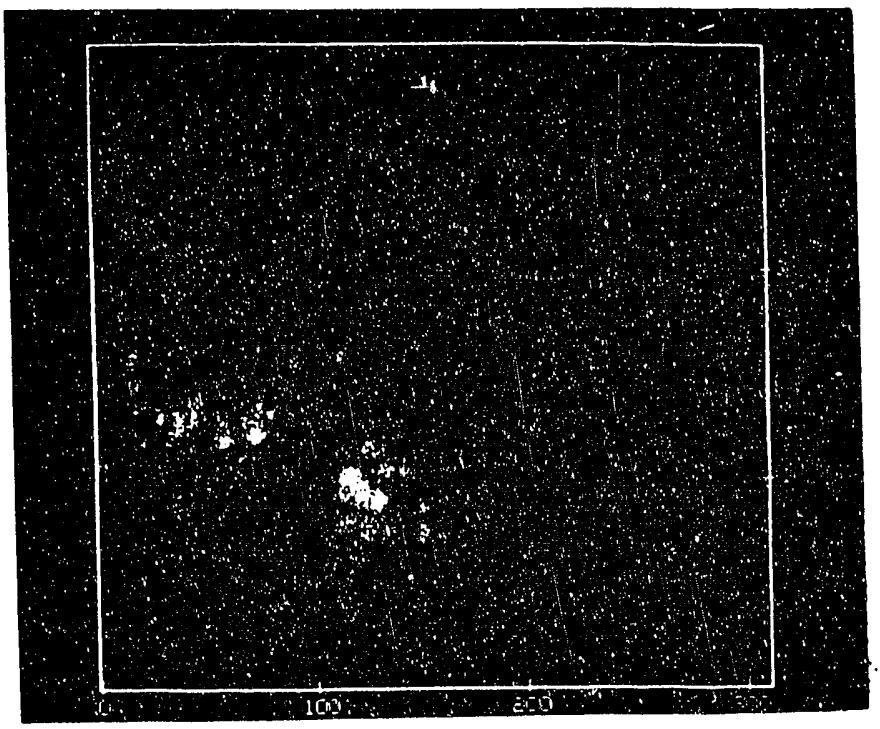

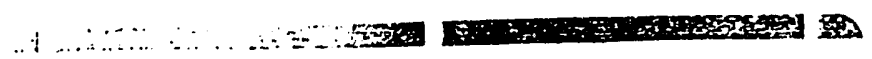

$$
\text { - } \because \cdots \equiv \text { (Siejel) - }
$$




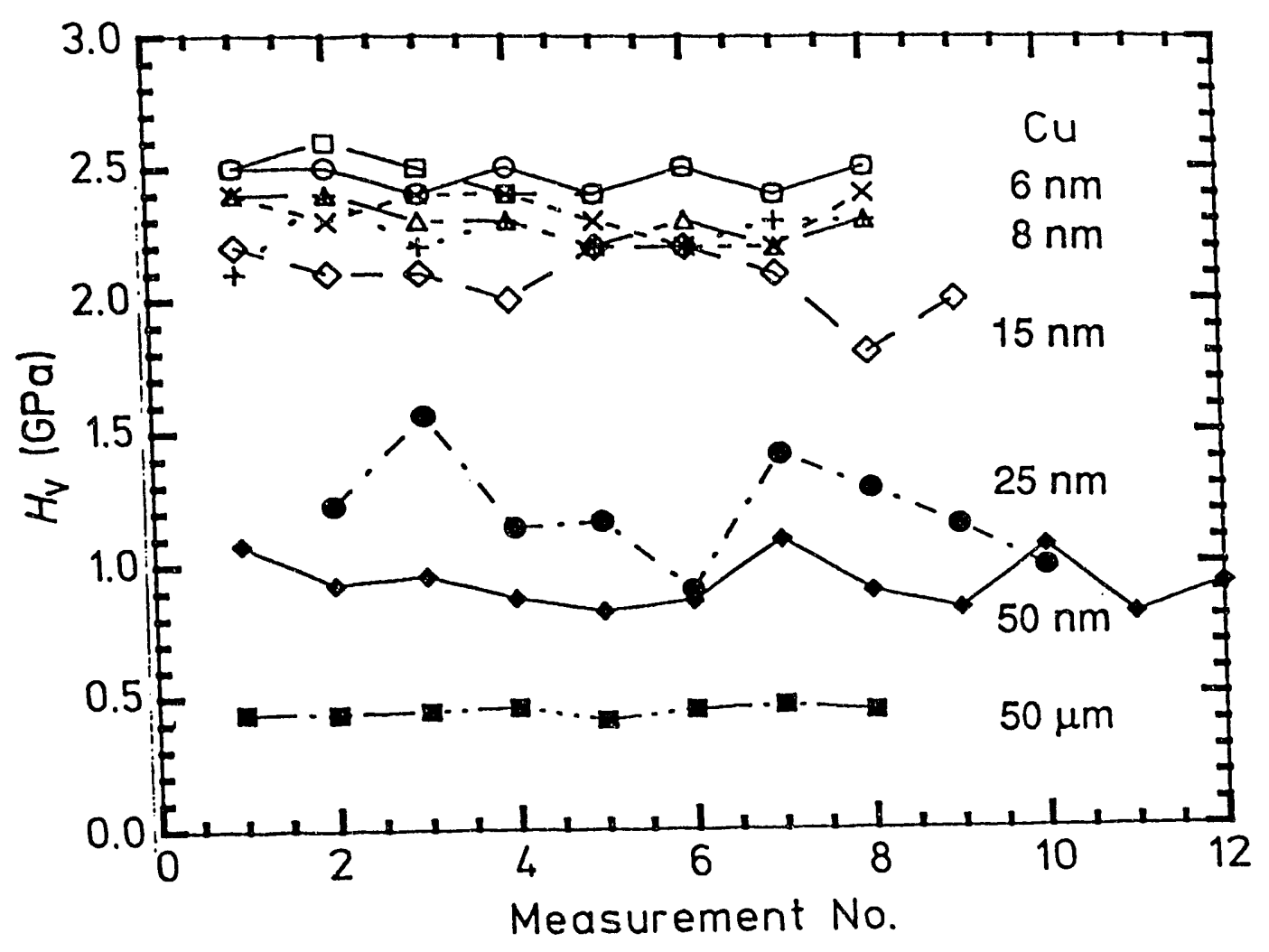

- Figure 4 (Siejel) 


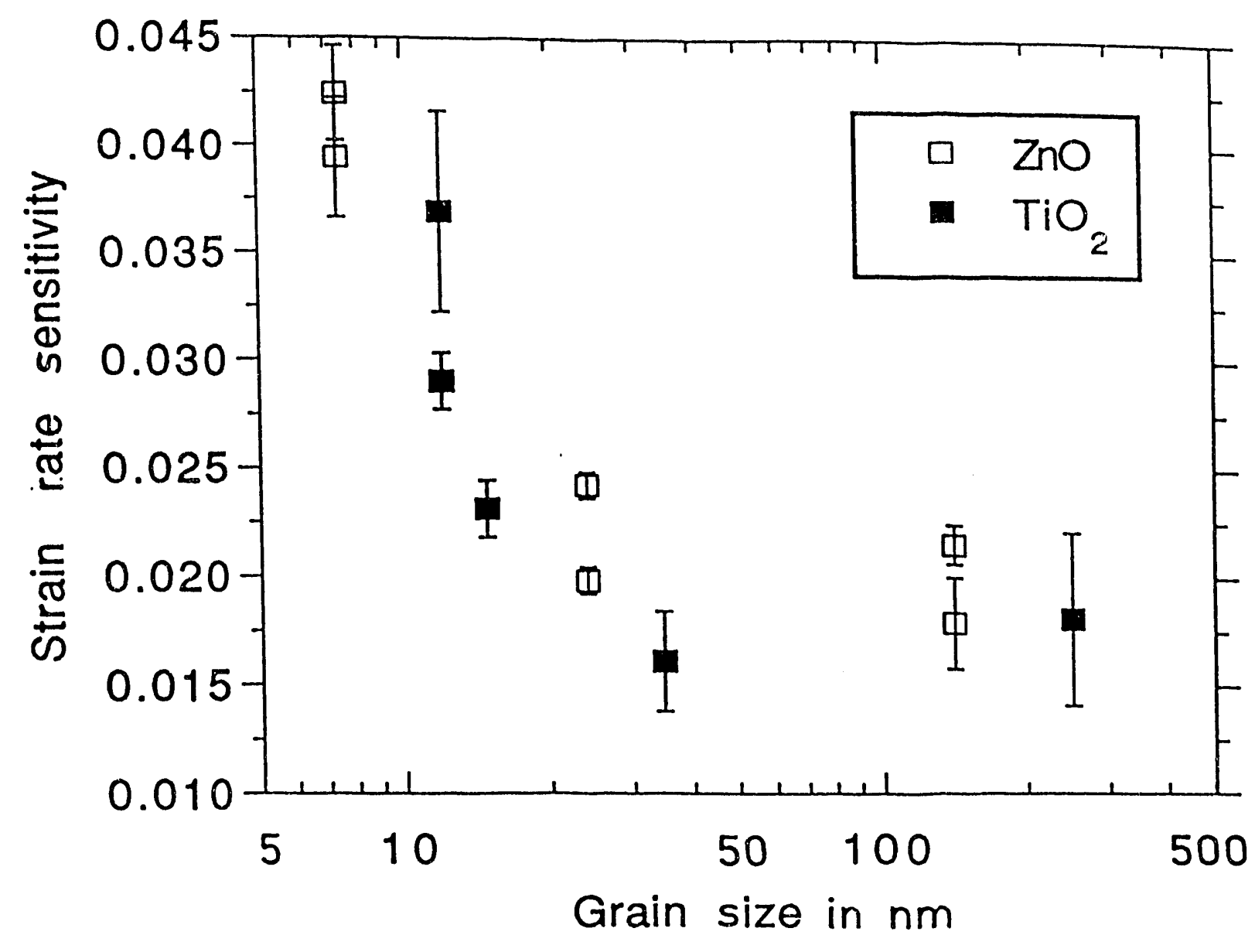

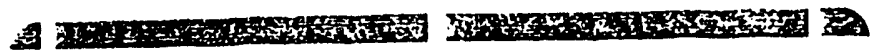

- Figure 5 (siejel) 


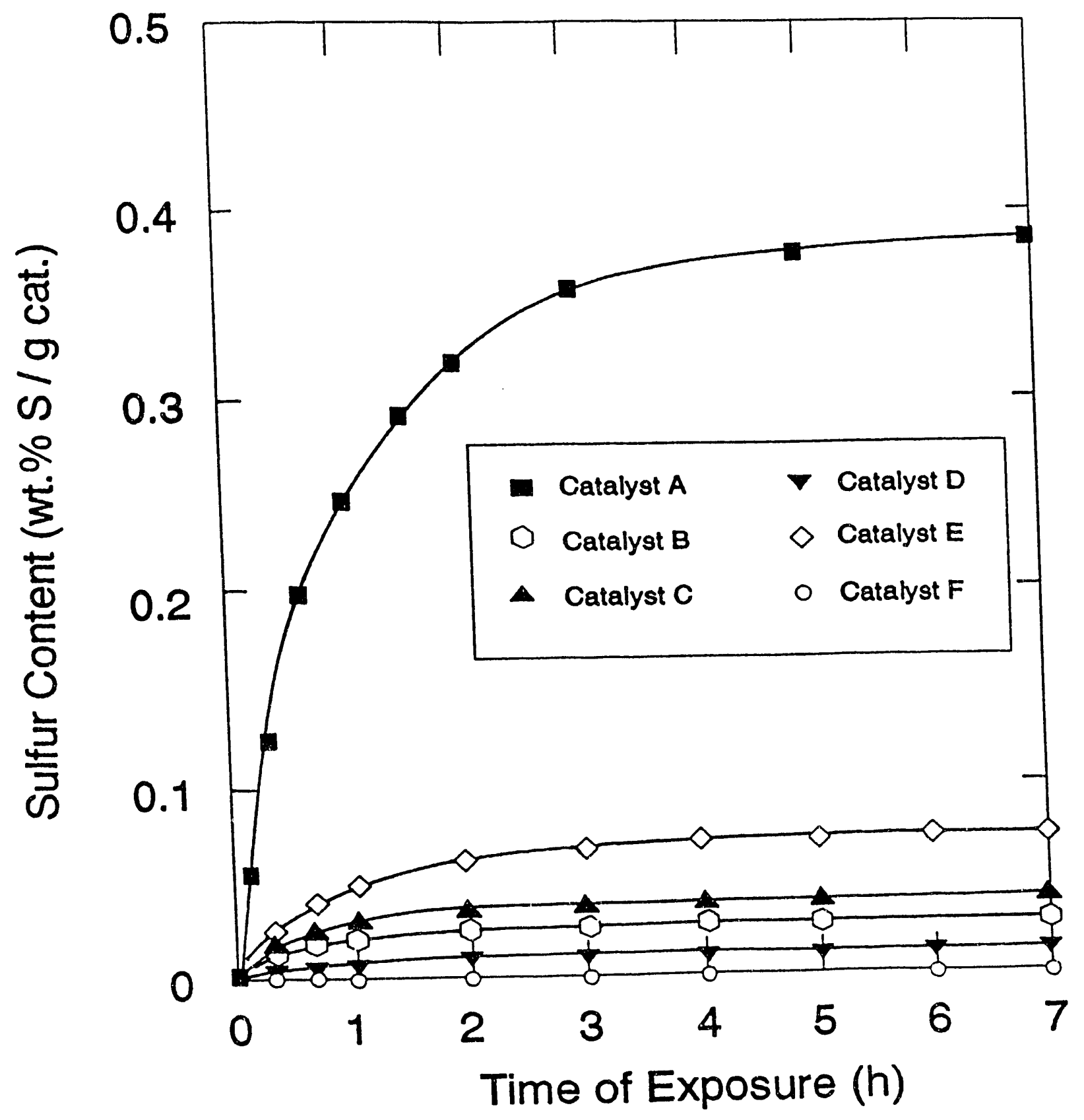

14

- Figure o (Steje!) 

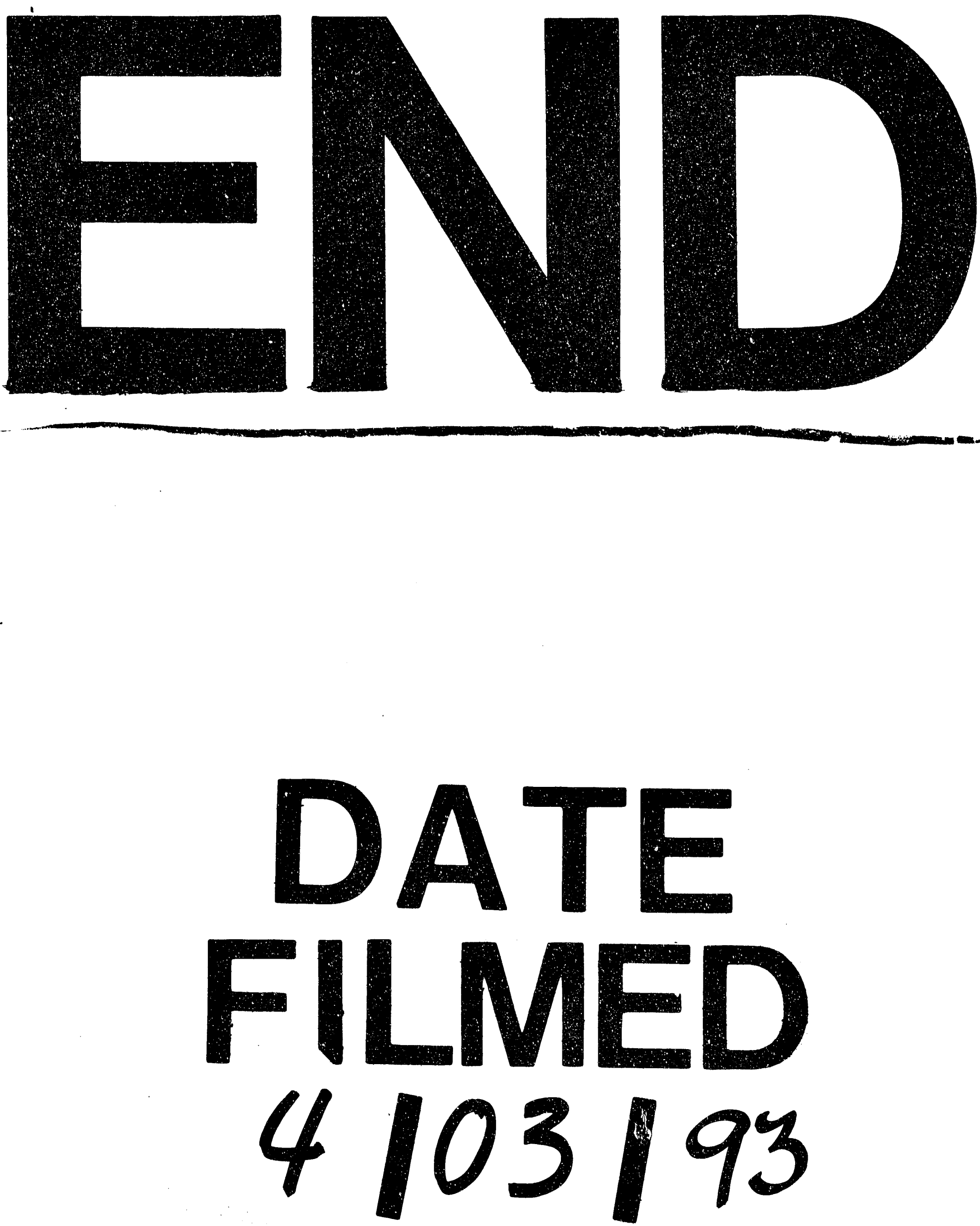
